EPJ Web of Conferences 29, 00036 (2012)

DOI: $10.1051 /$ epjconf/20122900036

(C) Owned by the authors, published by EDP Sciences, 2012

\title{
Domain wall dynamics of magnetically bistable microwires
}

\author{
V. Zhukova ${ }^{1 \mathrm{a}}$, J.M. Blanco ${ }^{2}$, M. Ipatov ${ }^{1}$ and A. Zhukov ${ }^{1,3}$ \\ ${ }^{1}$ Dpto. Fisica de Materiales, Fac. Quimicas, UPV/EHU, 20009 San Sebastian, Spain \\ ${ }^{2}$ Dpto. de Física Aplicada, EUPDS, UPV/EHU, 200018, San Sebastian, Spain \\ ${ }^{3}$ IKERBASQUE, Basque Foundation for Science, 48011 Bilbao, Spain
}

\begin{abstract}
We studied domain wall propagation of magnetically-bistable Fe- Co-rich microwires paying attention on effect of applied and internal stresses. We measured hysteresis loops and domain wall propagation in various magnetic $\mathrm{Fe}$ - Co-rich amorphous microwires with metallic nucleus diameters (from $12 \square \mathrm{m}$ till $22 \square \mathrm{m}$ ) using Sixtus Tonks-like experiments. Application of tensile stresses results in decreasing of domain wall velocity. We discussed magnetoelastic contribution in dynamics of domain wall propagation. We observed, that microwires with different geometries exhibit $v(H)$ dependences with different slopes. Application of stresses resulted in decrease of DW velocity, $v$, and DW mobility $S$. Quite fast DW propagation $(v$ till $2500 \mathrm{~m} / \mathrm{s}$ at $\mathrm{H}$ about $30 \mathrm{~A} / \mathrm{m}$ ) has been observed in low magnetostrictive magnetically bistable $\mathrm{Co}_{56} \mathrm{Fe}_{8} \mathrm{Ni}_{10} \mathrm{Si}_{110} \mathrm{~B}_{16}$ microwires. Consequently, we can assume that generally magnetoelastic energy affects DW dynamics: decreasing magnetoelastic energy, $K_{m e}$, DW velocity increases.
\end{abstract}

\section{Introduction}

Ferromagnetic glass coated thin wires (typically of 5-30 $\mu \mathrm{m}$ in diameter) exhibit unusual and interesting magnetic properties such as magnetic bistability and giant magneto-impedance, GMI, effect [1,2]. Magnetic bistability, observed previously in few amorphous materials, is related with single and large Barkhausen jump [2,3]. The characteristic feature of the magnetic bistability is the appearance of rectangular hysteresis loop. Such behavior was observed in wire shaped samples when the sample length and the applied magnetic field are above of some critical values [3,4]. Single Large Barkhausen Jump, observed in different families of amorphous wires has been interpreted as the magnetization reversal in a large single domain [2-5]. The rectangular hysteresis loop has been interpreted in terms of nucleation or depinning of the reversed domains inside the internal single domain and the consequent domain wall propagation [2-6]. Consequently, perfectly rectangular shape of the hysteresis loop has been related with a very high velocity of such domain wall propagation.

It is worth mentioning, that the domain wall (DW) propagation has been observed in different kinds of magnetic materials with rectangular hysteresis loop. Particularly, domain wall velocity has been successfully measured in conventional amorphous wires and Ni single crystals $[7,8]$.

\footnotetext{
a e-mail : arkadi.joukov@ehu.es
}

This is an Open Access article distributed under the terms of the Creative Commons Attribution License 2.0, which permits unrestricted use, distribution, and reproduction in any medium, provided the original work is properly cited. 
On the other hand, recently controllable and fast DW propagation observed in thin magnetic wires (planar and cylindrical) has been proposed for high density data storage devices (magnetic random memory MRAM devices, logic devices) $[9,10]$. Great efforts have been paid to increase the DW velocity of planar thin wire, considering its great importance for proposed applications [9].

It is worth mentioning, that generally much faster DW propagation at relatively low magnetic field has been reported for cylindrical thicker amorphous micrometric wires [2-6]. The micromagnetic origin of head-to head DW in microwires is still unclear, although it is clear that this DW is relatively thick and has complex structure [11,12]. At the same time it is commonly assumed, that the simultaneous solidification of composite microwire consisting of ferromagnetic metallic nucleus inside the glass coating introduces considerable residual stresses inside both ferromagnetic metallic nucleus and glass coating [13- 17]. But until now there are only few reports on the magnetoelastic contribution in DW dynamics of microwires [18].

Therefore, in this paper we are trying to reveal the effect of magnetoelastic anisotropy on DW propagation in amorphous magnetically bistable microwires.

\section{Experimental details}

Dependences of DW velocity on applied magnetic field have been measured by using modified Sixtus Tonks-like method, as described recently in ref. [19]. Particularly in order to activate DW propagation always from the other wire end in our experiment we placed one end of the sample outside the magnetization solenoid. Magnetic field, $\mathrm{H}$, is generated by single layered solenoid applying rectangular shaped voltage. Applied stresses have been applied during DW dynamics measurements. The applied tensile stress within the metallic nucleus and glass shell was calculated as described in Ref. [20],

$$
\begin{aligned}
& \sigma_{\mathrm{m}}=\mathrm{kP} /\left(\mathrm{kS}_{\mathrm{m}}+\mathrm{S}_{\mathrm{gl}}\right) \\
& \sigma_{\mathrm{gl}}=\mathrm{P} /\left(\mathrm{kS}_{\mathrm{m}}+\mathrm{S}_{\mathrm{gl}}\right)
\end{aligned}
$$

where $\mathrm{k}=\mathrm{E}_{2} / \mathrm{E}_{1}, \mathrm{E}_{\mathrm{i}}$ are the Young's module of the metal $\left(\mathrm{E}_{2}\right)$ and the glass $\left(\mathrm{E}_{1}\right)$ at room temperature, $\mathrm{P}$ is the applied mechanical load, and $\mathrm{S}_{\mathrm{m}}$ and $\mathrm{S}_{\mathrm{gl}}$ respectively are the cross sections of the metallic nucleus and glass coating. As stated elsewhere [17], the Young's modulus of the metal $\left(\mathrm{E}_{2}\right)$ and glass $\left(E_{1}\right)$ have roughly the following values: $E_{1}=7,5 \times 10^{10} \mathrm{~Pa}$ and $\mathrm{E}_{2}=1,5 \times 10^{11} \mathrm{~Pa}$.

We used 3 pick-up coils, mounted along the length of the wire and propagating DW induces electromotive force (emf) in the coils, as described in ref. [19].

Then, DW velocity is estimated as:

$$
v=\frac{l}{\Delta t}
$$

where $l$ is the distance between pick-up coils and $\Delta t$ is the time difference between the maximum in the induced emf.

We studied a number of amorphous Fe-Co based glass-coated microwires, produced by TaylorUlitovki method, as described elsewhere [3]. Studied microwires of $\mathrm{Co}_{56} \mathrm{Fe}_{8} \mathrm{Ni}_{10} \mathrm{Si}_{10} \mathrm{~B}_{16}$, $\mathrm{Co}_{41.7} \mathrm{Fe}_{36.4} \mathrm{Si}_{10.1} \mathrm{~B}_{11.8}, \quad \mathrm{Fe}_{55} \mathrm{Co}_{23} \mathrm{~B}_{11.8} \mathrm{Si}_{10.2}, \quad \mathrm{Fe}_{70} \mathrm{~B}_{15} \mathrm{Si}_{10} \mathrm{C}_{5}, \mathrm{Fe}_{72.75} \mathrm{Co}_{2.25} \mathrm{~B}_{15} \mathrm{Si}_{10}$ and $\mathrm{Fe}_{16} \mathrm{Co}_{60} \mathrm{Si}_{11} \mathrm{~B}_{13}$ compositions of ferromagnetic nucleus have positive magnetostriction constant and diameters of metallic nucleus from 2,8 till $22 \mu \mathrm{m}$. It is worth mentioning, that the magnetostriction constant, $\lambda_{\mathrm{s}}$, in system $\left(\mathrm{Co}_{\mathrm{x}} \mathrm{Fe}_{1-\mathrm{x}}\right)_{75} \mathrm{Si}_{15} \mathrm{~B}_{10}$ changes with $\mathrm{x}$ from $-5 \times 10^{-6}$ at $\mathrm{x}=1$, to $\lambda_{\mathrm{s}} \approx 40 \times 10^{-6}$ at $\mathrm{x} \approx 0.2$ [21].

Within each composition of metallic nucleus we also produced microwires with different ratio of metallic nucleus diameter and total diameter, $D$, i.e. with different ratios $\rho=d / D$. This allowed us to control residual stresses, since the strength of internal stresses is determined by ratio $\rho$ [13-17]. In this way we studied the effect of magnetoelastic contribution on DW dynamics controlling the magnetostriction constant, applied and/or residual stresses.

Hysteresis loops have been measured using vibrating sample magnetometer VSM. 


\section{Experimental results and discussion}

Hysteresis loops of few studied microwires $\left(\mathrm{Fe}_{70} \mathrm{~B}_{15} \mathrm{Si}_{10} \mathrm{C}_{5}\right.$ and $\left.\mathrm{Fe}_{72.75} \mathrm{Co}_{2.25} \mathrm{~B}_{15} \mathrm{Si}_{10}\right)$ with different metallic nucleus diameters and similar Fe-rich composition are shown in Fig. 1. As can be appreciated, considerable increasing of switching filed is observed when ferromagnetic metallic nucleus diameter decreases till $1,2 \mu \mathrm{m}$. At the same time, rectangular hysteresis loop shape is maintained even for smallest microwires diameters. Previously similar increase of coercivity with decreasing the metallic nucleus diameters have bee attributed to enhanced magnetoelastic energy arising from enhanced internal stresses when $\rho$-ratio is small [13-17]. Consequently, one of relevant parameters affecting strength of internal stresses and consequently magnetoelastic energy is $\rho$-ratio. Usually it is assumed that domain wall (DW) propagates along the wire with a velocity:

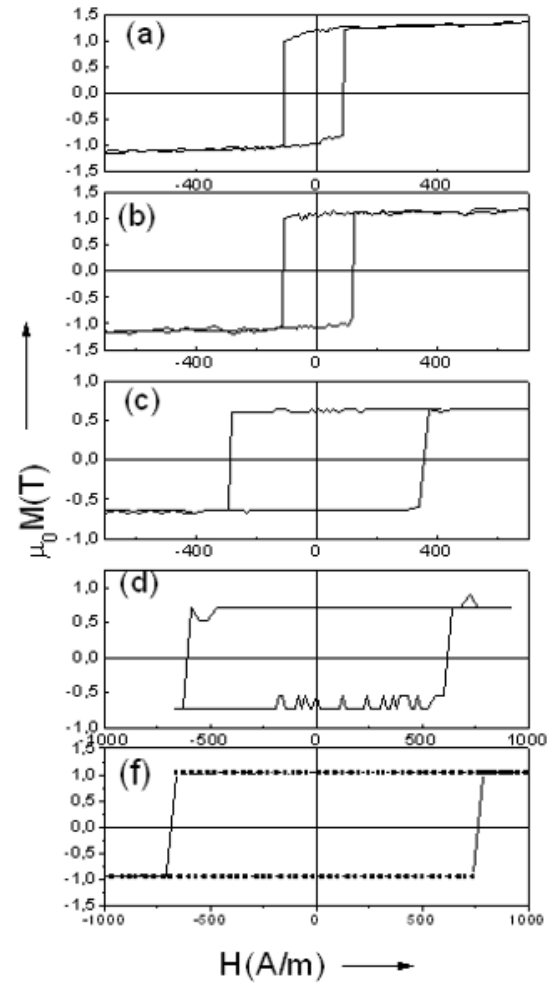

Fig. 1. Hysteresis loops of Fe-rich amorphous microwires with the same sample length and different metallic nucleus diameter $d$ and total diameters $D: F_{70} B_{15} S_{10} C_{5}$ microwires with $\rho=0.63 ; d=15 \mu \mathrm{m}$ (a); $\rho=0,48$; $d=$ $10,8 \mu \mathrm{m}$ (b); $\rho=0,26 ; d=6 \mu \mathrm{m}(\mathrm{c}) ; \rho=0,16 ; d=3 \mu \mathrm{m}$ (d) and of $\mathrm{Fe}_{72.75} \mathrm{Co}_{2.25} \mathrm{~B}_{15} \mathrm{Si}_{10}$ microwire with $\rho=0,14 ; \mathrm{d} \approx$ $1,4 \mu \mathrm{m} \mathrm{D} \approx 10 \mu \mathrm{m}$ (f).

$$
v=S\left(H-H_{0}\right)
$$

where $S$ is the DW mobility, $H$ is the axial magnetic field and $H_{0}$ is the critical propagation field.

In order to evaluate the effect of $\rho$-ratio, i.e. effect of residual stresses on DW dynamics, we performed measurements of $v(H)$ dependences in the microwires with the same composition, but with different $\rho$-ratios. Dependences of DW velocity on applied field for $\mathrm{Co}_{41.7} \mathrm{Fe}_{36.4} \mathrm{Si}_{10.1} \mathrm{~B}_{11.8}$ microwires with different ratios are shown on Fig.2.

On the other hand, magnetostriction constant is mostly determined by the metallic alloy composition. As mentioned above, the magnetostriction constant, $\lambda_{\mathrm{s}}$, in system $\left(\mathrm{Co}_{\mathrm{x}} \mathrm{Fe}_{1-\mathrm{x}}\right)_{75} \mathrm{Si}_{15} \mathrm{~B}_{10}$ changes from $35 \times 10^{-6}$ at $\mathrm{x}=0$ to $-5 \times 10^{-6}$ at $\mathrm{x}=1$ [21]. Consequently, magnetostriction constant, $\lambda_{\mathrm{s}}$, is determined by proportion of Co-Fe content in ferromagnetic alloy [21] and we can consider, that the strength of internal stresses is determined by the ratio $\rho=\mathrm{d} / \mathrm{D}$ [13-17]. Consequently we also 
measured $\mathrm{v}(\mathrm{H})$ dependences for two different microwire compositions with fixed $\rho$-ratio $(\rho=0,39)$ in order to evaluate effect of magnetostriction on $\mathrm{v}(\mathrm{H})$ dependence (Fig.3).

Analyzing Figs. 2,3 we can deduce, that at the same values of applied field, H, the domain wall velocity is higher for microwires with higher $\rho$-ratio, i.e. when the internal stresses are lower [1317].

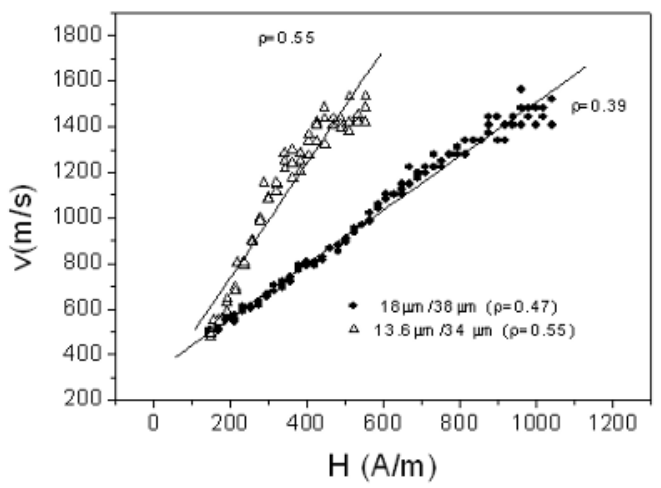

Fig.2. $\mathrm{v}(\mathrm{H})$ dependences for $\mathrm{Co}_{41.7} \mathrm{Fe}_{36.4} \mathrm{Si}_{10.1} \mathrm{~B}_{11.8}$ microwires with different ratios $\rho$.

It is worth mentioning, that the magnetoelastic energy, $\mathrm{K}_{\text {me }}$, is given by

$$
\mathrm{K}_{\mathrm{me}} \approx 3 / 2 \lambda_{\mathrm{s}} \sigma \text {, }
$$

where $\sigma=\sigma_{\mathrm{i}}+\sigma_{\mathrm{a}}$ - total stress, $\sigma_{\mathrm{i}}$-are the internal stresses, $\sigma_{\mathrm{a}}-$ applied stresses and $\lambda_{s}$ magnetostriction constant [14 ]. Dependences of domain wall velocity, $v$, on magnetic field, $\mathrm{H}$ for $\mathrm{Fe}_{16} \mathrm{Co}_{60} \mathrm{Si}_{13} \mathrm{~B}_{11}$ and $\mathrm{Co}_{41.7} \mathrm{Fe}_{36.4} \mathrm{Si}_{10.1} \mathrm{~B}_{11.8}$ amorphous microwires with the same $\rho$-ratio are shown in Fig.3. In this case, the effect of only magnetostriction constant is similar to that observed in Fig.2: higher magnetostriction (in according to ref. [17] for $\mathrm{Co}_{41.7} \mathrm{Fe}_{36.4} \mathrm{Si}_{10.1} \mathrm{~B}_{11}$ microwire $\lambda_{s} \approx 30 \times 10^{-6}$ should be considered, while for $\mathrm{Co}_{41.7} \mathrm{Fe}_{36.4} \mathrm{Si}_{10.1} \mathrm{~B}_{11.8}$ composition $\lambda_{s} \approx 30 \times 10^{-6}$ ) results in smaller DW velocity at the same magnetic field and smaller DW mobility, S.

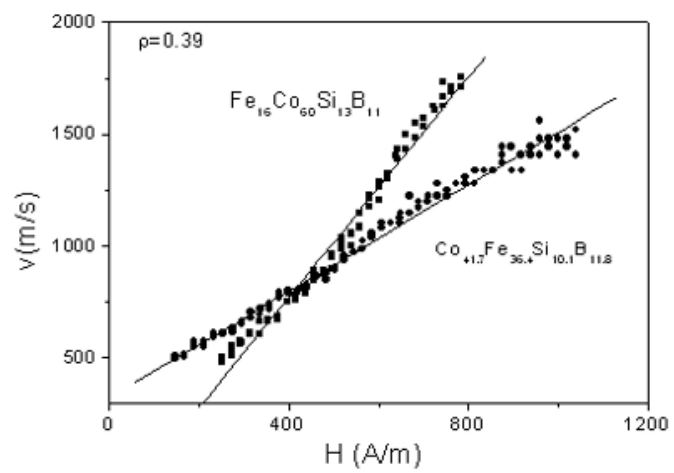

Fig.3. $v(\mathrm{H})$ dependences for $\mathrm{Fe}_{16} \mathrm{Co}_{60} \mathrm{Si}_{13} \mathrm{~B}_{11}$ and $\mathrm{Co}_{41.7} \mathrm{Fe}_{36.4} \mathrm{Si}_{10.1} \mathrm{~B}_{11.8}$ microwires with $\rho=0,39$.

Another way to change the magnetoelastic energy is the application of stresses during measurements. Consequently we measured $v(H)$ dependences under applied stress.

As can be observed from Fig.4, increasing of magnetoelastic energy, $\mathrm{K}_{\mathrm{me}}$, through application of stresses results in decreasing of DW velocity in $\mathrm{Co}_{41.7} \mathrm{Fe}_{36.4} \mathrm{Si}_{10.1} \mathrm{~B}_{11.8}$ microwires. Consequently, similarly with previous cases, magnetoelastic energy significantly affects $v(H)$ dependences.

Consequently, one of possibilities to enhance the DW velocity is to employ the microwires with smallest magnetostriction constant. Therefore we selected the $\mathrm{Co}_{56} \mathrm{Fe}_{8} \mathrm{Ni}_{10} \mathrm{Si}_{10} \mathrm{~B}_{16}$ amorphous microwires $\left(\lambda_{s} \approx 10^{-7}\right)$ for analysing the DW dynamics. As can be appreciated form Fig.5, the DW 
velocity values achieved in this microwire at the same values of applied field, is considerable higher (almost twice), than observed for microwires with higher magnetostriction constant (see Figs.2,3). Additionally, stronger effect of applied stresses on $\mathrm{v}(\mathrm{H})$ in $\mathrm{Co}_{56} \mathrm{Fe}_{8} \mathrm{Ni}_{10} \mathrm{Si}_{10} \mathrm{~B}_{16}$ amorphous microwires might be related with magnetostriction dependence on applied stresses, as described elsewhere [21]. In this case, since magnetostriction constant is low, stress effect on magnetostriction can be comparable with the magnetostriction value.

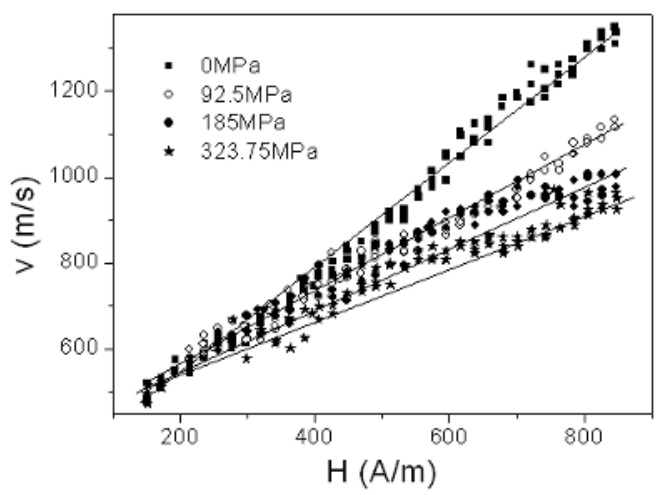

Fig. 4. Magnetic field dependences of DW velocity for $\mathrm{Co}_{41.7} \mathrm{Fe}_{36.4} \mathrm{Si}_{10.1} \mathrm{~B}_{11.8}$ microwires $(\rho=0,55)$ measured at different applied stresses.

It is worth mentioning that observed by us DW velocity values exceeds estimated maximum velocity (Walker limit) for DW propagation, which can be estimated from:

The domain wall dynamics in viscous regime is determined by a mobility relation (2), where $S$ is the domain wall mobility given by:

$$
S=2 \mu_{0} M_{\sigma} / \beta
$$

where $\beta$ is the viscous damping coefficient, $\mu_{0}$ is magnetic permeability of vacuum. Damping is the most relevant parameter determining the domain wall dynamics. Usually two contributions to viscous damping $\beta$ have been considered and generally accepted [6].

The first $\left(\beta_{e}\right)$ is determined by the micro-eddy currents circulating nearby moving domain wall. However, the eddy current parameter $\beta_{e}$ is considered to be negligible in high-resistive materials, like amorphous microwires, which additionally have quite thin diameters.

The second generally accepted contribution of energy dissipation is magnetic relaxation damping, $\beta_{r}$, related to a delayed rotation of electron spins. This damping is related to the Gilbert damping parameter, $\alpha$ and is inversely proportional to the domain wall width $\delta w$,

$$
\beta_{r} \approx \alpha M_{s} / \gamma \delta_{w} \approx M_{s}\left(K_{m e} / A\right)^{1 / 2}
$$

where $\gamma$ is the gyromagnetic ratio, $A$ is the exchange stiffness constant, $K_{m e}$ is the magnetoelastic anisotropy energy given by (5).

Consequently, the domain wall mobility, $\mathrm{S}$, can be affected by the magnetoelastic energy, $K_{m e}$, as we experimentally observed in few Co-Fe-rich magnetically bistable microwires (Figs, 2-5). 


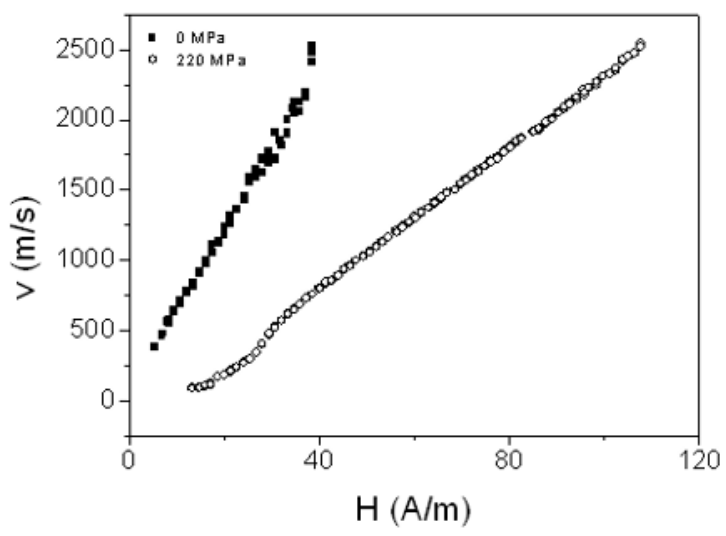

Fig.5. $v(\mathrm{H})$ dependences for $\mathrm{Co}_{56} \mathrm{Fe}_{8} \mathrm{Ni}_{10} \mathrm{Si}_{10} \mathrm{~B}_{16}$ microwires measured under application of applied stresses, $\sigma_{\text {app }}$.

It is interesting, that DW velocity observed in thinnest measured $\mathrm{Fe}_{72.75} \mathrm{Co}_{2.25} \mathrm{~B}_{15} \mathrm{Si}_{10}$ amorphous microwire with metallic nucleus diameters of $2,8 \mu \mathrm{m}$ is significantly lower and magnetic field at which DW propagation is observed in considerably higher than for thicker microwires (Fig.6). This fact can be particularly explained by significantly lower $\rho$-ratio $(\rho \approx 0,31)$ of this sample, i.e. by elevated residual stresses in such microwire.

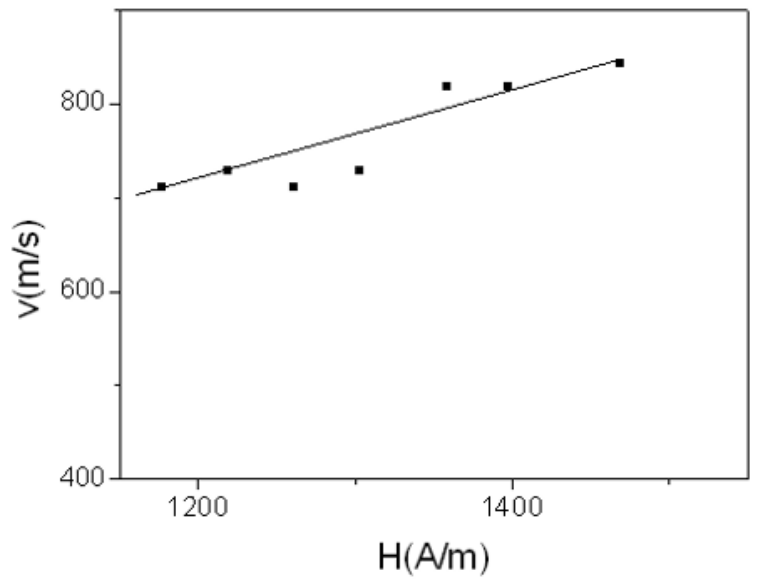

Fig.6. v(H) dependence for $\mathrm{Fe}_{72.75} \mathrm{Co}_{2.25} \mathrm{~B}_{15} \mathrm{Si}_{10}$ amorphous microwire with metallic nucleus diameter, d, of $2,8 \mu \mathrm{m}$ and total diameter $\mathrm{D} \approx 9 \mu \mathrm{m}$.

Resuming, from observed experimental dependences we can suggest, that DW dynamics in cylindrical microwires correlates with the magnetoelastic anisotropy and can be enhanced minimising the magnetoelastic anisotropy.

\section{Conclusions}

We experimentally observed that the magnetoelastic energy significantly affected domain wall dynamics in magnetically bistable microwires. Considering aforementioned we assume that in order to achieve higher DW propagation velocity at the same magnetic field and enhanced DW mobility special attention should be paid to decreasing of magnetoelastic energy. Applied and internal stresses result in decreasing of DW velocity. 


\section{Acknowledgements}

This work was supported by EU ERA-NET programme under project "SoMaMicSens" (MANUNET-2010-Basque-3), by Spanish Ministry of Science and Innovation, MICINN under project MAT2010-18914 and by the Basque Government under Saiotek 09 MICMAGN project (SPE09UN38).

\section{References}

1. D. C. Jiles, Acta Materialia,. 51, 5907 (2003).

2. V. Zhukova, M. Ipatov and A Zhukov, Sensors 9, 9216 (2009)

3. A.P. Zhukov, Materials and Design, 5, 299 (1993)

4. A. Zhukov, Appl. Phys. Let. 78, 3106 (2001).

5. A. P. Zhukov, M. Vázquez, J. Velázquez, H. Chiriac and V. Larin, J. Magn and Magn, Mater $151132(1995)$

6. R. Varga, A. Zhukov, V. Zhukova, J. M. Blanco and J. Gonzalez, Phys. Rev. B 76, 132406 (2007).

7. W. Riehemann and E. Nembach, J. Appl. Phys. 55,1081(1984)

8. D.-X. Chen, N. M. Dempsey, M. Vázquez and A. Hernando, IEEE Trans. Magn. 31, 781 (1995).

9. M. Hayashi, L. Thomas, Ch. Rettner, R. Moriya, X. Jiang, and S. Parkin, Phys.Rev. Lett. 97, 207205 (2006)

10. D. A. Allwood, G. Xiong, C. C. Faulkner, D. Atkinson, D. Petit, and R. P. Cowburn, Science 309, No 5741, 1688 (2005).

11. S. A. Gudoshnikov, Yu. B. Grebenshchikov, B. Ya. Ljubimov, P. S. Palvanov, N. A. Usov, M. Ipatov, A. Zhukov, and J. Gonzalez, Phys. Status Solidi A 206 613-617 (2009)

12. P. A. Ekstrom and A Zhukov, J. Phys. D: Appl. Phys. 43205001 -6 (2010)

13. H. Chiriac, T. A. Ovari, and Gh. Pop, Phys. Rev. B 42 10105-10113 (1995)

14. A.Zhukov and V. Zhukova, "Magnetic properties and applications of ferromagnetic microwires with amorphous and nanocrystalline structure", Nova Science Publishers, Inc. 400 Oser Avenue, Suite 1600 Hauppauge, NY 11788, 162 p. ISBN: 978-1-60741-770-5(2009).

15. H. Chiriac, T.-A- Ovari and A. Zhukov, J. Magn. Magn. Mater. 469 254-255 (2003)

16. A S Antonov, V T Borisov, O V Borisov, A F Prokoshin and N A Usov, J. Phys. D: Appl. Phys. $331161(2000)$.

17. J. Velázquez, M. Vazquez and A. Zhukov, J. Mater. Res.11 No10 2499 (1996)

18. J.M. Blanco, V. Zhukova, M. Ipatov, and A Zhukov, Phys. Status Solidi A 208, No. 3, 545 (2011)

19. M. Ipatov, V. Zhukova, A. K. Zvezdin and A. Zhukov, J. Appl. Phys. ,106, 103902 (2009)

20. A. Zhukov, Adv. Func. Mat., 16, 5, 675 (2006)

21. H. Fujimori, K. I. Arai, H. Shirae, H. Saito, T. Masumoto and N. Tsuya, Japan J. Appl. Phys. 15 No 4, 705 (1976) 